\title{
A Phenomenological Study on the Quality of Life Among Patients with Osteoarthritis Admitted for Rehabilitative Physiotherapy in a Private Hospital in Kuala Lumpur
}

\author{
R. (III) P. DIOSO ${ }^{1 *}$ AND R.TANGGAYA ${ }^{2}$
}

\begin{abstract}
The aim of this study is to explore patients' experiences and how their rehabilitation and physiotherapy at a private hospital in Kuala Lumpur, Malaysia has affected their quality of life (QOL) due to osteoarthritis (OA). In addition, this study also aims to explore patients' perspectives of the rehabilitation and physiotherapy for their OA before and after which affects moreover their QOL. The seven domains of the QOL were the physiological, social, financial, environmental, psychological, level of independence and spiritual - aimed to guide the theme of the interview sessions.

A qualitative design was used. Of the 50 patients purposively enrolled only six were selected using inclusion and exclusion criteria. Thematic analysis addressed the seven domains of the QOL for data analysis. A 60-minutes interview session was audio-recorded.

The rehabilitation therapy was explored on all seven domains of the QOL. Of the six respondents four explored their lived experiences on the social and environmental domain, while five on the physiologic domain. The spiritual, psychological, level of independence and financial domains were mostly explored by the six respondents that have affected their QOL.
\end{abstract}

Key words: Quality of life; physiotherapy; qualitative phenomena, rehabilitation therapy, osteoarthritis

The aim of this study is to explore patients' experiences and how their rehab at a private hospital in peninsular Malaysia has affected their quality of life (QOL) due to osteoarthritis (OA). In addition, this study also aims to explore patients' perspectives of the rehabilitation and physiotherapy which affects moreover their QOL.

The purpose of this study is to establish a concept of health promotion for other patients with OA, as it opens avenues on how to improve their QOL. It is also hoped that this study will help promote health-related strategies for healthcare professionals on how to advise OA patients in improving their QOL.

The QOL according to the World health Organization (WHO 1998) measures seven domains - physiologic, psychologic, environmental, social, level of independence, financial and spiritual. It is a descriptive term that refers to the functional abilities of a person related to the ordinary tasks of daily living (Fallowfield 2009).

An OA, on the other hand is known as the degenerative arthritis or degenerative

\footnotetext{
${ }^{1}$ Faculty of Nursing, Lincoln University College, Malaysia

${ }^{2}$ Physiotherapist, Yayasan Sultan Idris Shah, Ipoh, Malaysia

* Corresponding author (e-mail: duke@lincoln.edu.my)
} 
joint disease, which are group of mechanical abnormalities relating to degradation of joints, including articular cartilage and subchondral bone that has an effect on a patient's QOL (Coggon et al. 2001; Brooks 2002; Li et al. 2013).

Physiotherapy as a rehabilitative treatment for OAs can improve their QOL (Brooks 2002). They commonly involve active range of motion exercises and massage (Brooks 2002; Coggan et al. 2001). Rehabilitation of OAs in addition to physiotherapy sessions, in order to achieve QOL in its broadest sense commonly involves a combination of self-performed exercises, lifestyle modification, and analgesics (Bosomworth 2009). If pain is not reduced, debilitation or joint replacement surgery may be used to improve their QOL (Tortora \& Derrickson 2010; Felson 2004).

\section{BACKGROUND}

In Malaysia, a study has shown that patients with OAs attending primary care clinics have relatively poor QOL pertaining to the physical domain and its health-related components (Zakaria et al. 2009). In addition, analgesics in Malaysia are recommended to ensure pain free QOL with the full four weeks of check-up with treatment (Chin et al. 2012). This makes regular visits to physiotherapy department impossible so, a self-management treatment is cost-effective and a coping management which can improve their working life more qualitatively (Felson 2004).

It will be beneficial to undertake this study since there is not enough research and/ or literatures published in Malaysia to explore the lived experiences of patients with OAs that affects their QOL. However, reviewing similar concepts are also necessary in order to substantiate this research.

\section{CONCEPT}

The environmental domain is conceptualized by using interventions that comprises a series of highlighted daily activities such as chores, as well as leisure. The domain of the patients' level of independence with OAs is conceptualized as the ability to cope with daily routine especially with jobs (Power et al. 2008; Rosemann et al. 2008/2005). Power et al. (2008) also added that QOL measures OA's severity (the physiologic domain), depression (the social domain) and fatigue (the psychological domain). Fujita et al. (2006) says that the physiologic domain is measured by disease progression and treatment. The spiritual domain is measured by the thoughts on OA and their forgiveness and blame for acquiring the illness, and how they accept the reality that they have OA for life. Lastly, the financial domain measures the employability of an individual and how they

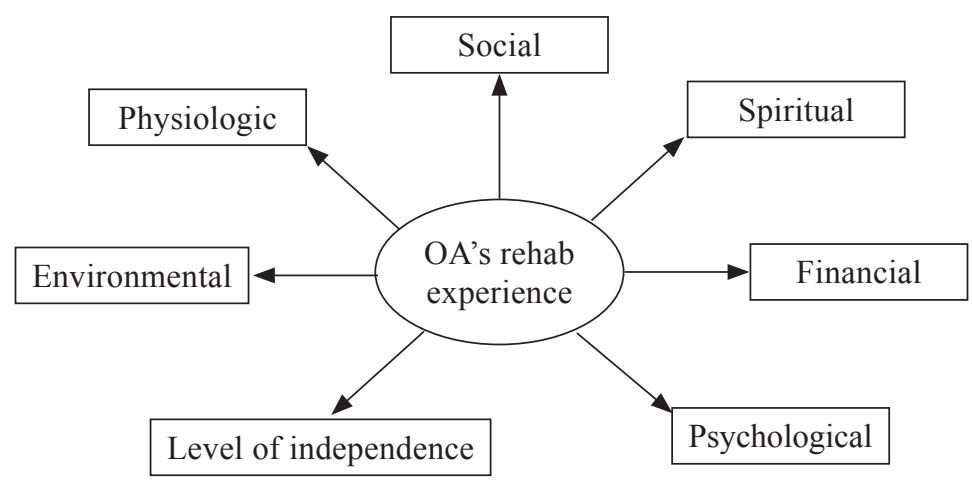

Figure 1. The concept of the study. 
keep their employment status to maintain their financial needs even with OA (Abdullah et al. 2013).

The reviewed concepts will help formulate strategies on how to explore patients' QOL with OA. Figure 1 shows the concept of the study.

\section{LITERATURE REVIEW}

Four literatures inspired this study and also helped in the formulation of the concept.

The study of Morden et al. (2014) is the first literature reviewed, that used a guide book in planning for the rehabilitation as a form of intervention. The concept of this study explored how patients used the guidebooks in managing their OAs to achieve a QOL.

The findings from the study of Morden et al. (2014) were divided into groups - the interview results from the healthcare practitioners and patients. The result of the interview from the healthcare practitioners were further divided into themes - the perceptions on benefits of patients and the managing consultations. The healthcare practitioners gave positive answers on the themes that explored the good benefits of the guidebook and how it is easily understood by the patients with OAs. While the result of the interview to the patients also gave a positive remark on the guidebook by answering the interview saying that it clarifies and explains OAs well and it provides positive aspects of biomedical information therefore it is very useful on self-management even if drugs are included in the manual.

In addition, the study of Morden et al. (2014) is very appropriate since the concept of this study supported self-management to achieve a QOL. Eight subjects in the West Midlands and North West of England were recruited to take part: four control practices and four intervention practices. Semi-structured interviews were undertaken with patients $(n=29)$, general practitioners $(n=9)$ and practice nurses $(n=4)$ which was a very appropriate sampling size in a focus group design that was divided into three groups in order to explore experiences of the subject with the use of the OA guidebook. Morden et al. 2014 analyzed the data using thematic analysis which was also very appropriate for this study addressing the seven domains of the QOL.

The questions adapted from the study of Morden et al. (2014) will focus on the physiologic, environmental and level of independence domain with questions enumerated as:

(1) Engaging in some form of selfmanagement, either self-learned from experience or disseminated via social networks (physiologic);

(2) Continuing existing physical activities without worry about damaging joints or causing additional pain (level of independence); and

(3) Working with confidence that all forms of environments are safe for them to work and/or perform their activities of daily living (environmental).

However, the authors (in this case the reviewers) must take caution because the sample of patients in the study of Morden et al. (2014) were all white British. Other ethnic groups would have explored the guidebook on OAs differently due to cultural diversity. Therefore, the physiologic, environmental and level of independence domains in this phenomenological study must be explored appropriately for the Malaysian ethnic groups in the selected private hospital in Kuala Lumpur.

However, the study of Morden et al. (2014) did not consider the social and psychological domains of the QOL. 
This is why the study of Power et al. (2008) was selected as the second literature to be reviewed because it focuses on fatigue which is very appropriate and useful for the concept of this phenomenological study. In addition, the study of Power et al. (2008) was very appropriate to enlighten this research since we (the authors) aim to explore the patients' rehabilitation experiences.

The findings on the study of Power et al. (2008) were divided into five themes - fatigue characteristics, factors affecting fatigue, impact of fatigue, methods of coping with fatigue and discussion about fatigue with significant others. The subjects with OAs explored the use of exercise and how to control pain by adhering to pain medications may eventually avoid depression - the social and psychological domain.

Power et al. (2008) used audio tapes and transcribed verbatim results which also inspired this study because it is less complex if subjects' answers were audio recorded.

The advantage of the study of Power et al. (2008) from the study of Morden et al. (2014) was the highlighted emotional consequences and impacts on daily activities such as chores, as well as leisure and social activities. However, both studies did not explore experiences with OAs post-arthroplasty surgery that explored forgiveness and blame of the subjects addressing the spiritual domain of the QOL.

This is why the Fujita et al. (2006) was selected to be the third literature to be reviewed. Fujita et al. (2006) used a semi-structured interview session 48 hours after and before total hip arthroplasty (THA) as a surgical management for patients with OA.

Lastly, the content of the questions set by Fujita et al. (2006) can also be useful in the thematic content of this research's questionnaire.

The questionnaires for the interview sessions were basically enumerated as:

i. Experience with OA prior to THA, focusing on disease progression and treatment, and the impact of OA on the participant's life (physiologic)

ii. The reason for undergoing the surgical procedure and the situation the participant was in at the time (psychological); and

iii. The participant's thoughts on OA affecting their spiritual life after surgery (spiritual).

Again and again, adapting the physiologic domain from the study of Fujita et al. (2006) must be explored with caution that is appropriate with the Malaysian ethnic group and culture since it is mostly affected by such (Fallowfield 2009; Tortora \& Derrickson 2010).

However, Fujita et al. (2006), Power et al. (2008) and Morden et al. (2014) did not consider the financial domain that affects the QOL among patients with OA.

The financial domain according to the study that was done by Abdullah et al. (2013) measures the employability of an individual and how they keep their employment status to maintain their financial needs.

The study done by Abdullah et al. (2013) was the fourth literature selected to be reviewed because it used a focused group who are obese and they eventually led to OA affecting their finances. A focused group will be very essential to enlighten the concept of this research because the questionnaires with contents that can be used for the interview involves the financial statuses of patients with OAs. 
The findings of Abdullah et al. (2013) on the interviewees' perceptions and their attitudes towards their experiences on OAs for 20 years, was explored to have affected their financial domain from past to present. Abdullah et al's (2013) interview sessions were recorded, transcribed and coded. Their discussions centered around six themes. The financial domain focuses on these questions:

Q1: How do you feel about your financial expenditures having daily knee pain?

Q2: How has having knee pain interfered with your financial life?

Q3: From what we have discussed so far, what bothers your financial needs the most about having knee pain?

Q4: Is any member of your family affected by you having knee pain?

Q5: What do you personally do to deal with your knee pain while working?

Q6: How do you cope with your knee pain when doing your daily activities?

Moreover, the four selected studies will help this research design its methodology and interview approach.

\section{METHODOLOGY}

This section commences by addressing the design of this research. The description of the setting, subjects, and methods of data collection follows. The method of developing the instruments and how to analyse the data collected will also be explained. Finally, this section ends by explaining the ethics that was considered.

\section{Research Design}

A phenomenological qualitative design was the most appropriate to be used in this study in order to achieve the aims and purposes set.
The research design can be further divided into setting and subject designs in order to strategically discuss the cause-effect variables (Groves et al. 2009).

The effect variable was the patients' experiences on the QOL that this research deemed to explore while the causative variable was the rehabilitation process (Figure 1).

Setting. One of the reasons why it was best to conduct an interview in Peninsular Malaysia for the setting was because some of the lived experiences to be asked to the participants were in English and it was difficult to translate it in the Malay language. In addition, most of the prospective subjects in peninsular Malaysia are well versed in communicating spoken English (Ministry of Higher Education 2010). Therefore the medium of communication in this particular setting was mostly in English or in Bahasa Melayu (the two major languages in peninsular Malaysia). Other languages that are prominently spoken in peninsular Malaysia such as Mandarin and Cantonese (Chinese), Tamil and Hindi (Indian) are not used as the medium of communication.

Secondly, the reason why the private hospital was selected as the setting was because subjects with OAs interviewed had a salary rate ranging from RM 3000 and above (Chin et al. 2012; Blixen \& Kippes 1999). These patients (with salary rate of RM 3000 and above) usually experiences difficulties in paying their bills (Chin et al. 2012; Blixen \& Kippes 1999) since OAs was a long-term treatment which requires expensive drugs that is likely to affect their QOL (Zakaria et al. 2009). Lastly, the hospitals in peninsular Malaysia as a setting can be a safe place to conduct an interview among OAs patients since emergency equipment are more accessible if life threatening situations occurs that can be triggered because of the interview sessions (Wolfey \& Holley 1997; Roos \& Larsen 2003). 
In addition, a private owned hospital found in the state of Kuala Lumpur are more open for research (Zakaria et al. 2009; Chin et al. 2012; Li et al. 2013). The prospective subjects confined in private-owned hospitals in particular do not require too much negotiation with researchers unlike in public hospitals where managements and/or managers do not want researchers to explore and disclose their patients' illnesses (Chin et al. 2012).

Therefore selecting prospective subjects for this study are easier in a private hospital in peninsular Malaysia.

Subjects. Purposive sampling was used to select the prospective subjects to be interviewed. This sampling technique was deemed best to help the researchers find a small sample size which is accessible for an in-depth interview and whose findings can be generalized to the accessible population rather than the complete target population (Denzin \& Lincoln 2005; p. 23; Saldana 2012; p. 74). Inclusion and exclusion criteria are required in a purposive sampling technique (Saldana 2012).

Patients had to be suffering from OA - a doctor's diagnosis must be reflected in their patients' record - as one of the inclusion criteria. The patients also have to have registered in the past with the physiotherapy outpatient department in the specific privateowned hospital found in Kuala Lumpur between 2013 to present; whether they have undergone surgical treatments or still on the rehabilitation process. In addition, exploring patients' lived experiences with physiotherapy to achieve a QOL can be more multi-dimensional since they are from the state of Kuala Lumpur where lifestyles are diverse (Zakaria et al. 2009).

Another inclusion criterion requires from the prospective subjects was to be able to orally communicate in English well and could express their answers to the interviewer in spoken English.
Lastly, only adult patients ages 21 and above were included.

The first exclusion criteria on the other hand were pediatrics aged 18 years old and below. This is because the aetiology of OAs is often seen on adult age (Dias et al. 2003; Jakobsson \& Hallber 2002). In addition, QOL is best explored among adults ages 21 years old and above with OA. Therefore patients who are below 20 years old are also excluded since they have lesser QOL domains to explore (Wolfey \& Holley 1997).

Secondly, patients who cannot express themselves in spoken English were also excluded especially those patients with OAs who were born mute and could only use handsign languages since the interview session would audio-tape their answers making sign languages inappropriate. Plus even if the sign languages could be translated into verbal, the vocal expressions and intonations would not be originally coming from the prospective subjects and might lead to a misinterpretation of the desired answers from the subjects. In addition, patients who are disoriented such as those who are old, ages 70 years old and above and are not anymore capable of using intellectual reasoning (Dias et al. 2003) are excluded since most of them cannot give an answer.

Thirdly, patients with OAs who had sought consultations in their community health clinics or in village hospitals are excluded since these patients rarely expresses themselves in spoken English (Zakaria et al. 2009) which this research requires. Again and again, translating their answers from other languages or dialects might still cause misinterpretation.

Lastly, foreigners who were not capable of expressing conversational Bahasa Melayu were excluded since some questions regarding the domains of the QOL had cultural Malaysian terminologies (words) which were difficult to 
be translated in English or have no equivalent English words.

Of the 50 patients enrolled, only six subjects were selected to be interviewed. Figure 2 shows how the 44 enrolled participants were eliminated based on the exclusion criteria.

\section{Data Collection}

The six participants selected, were primarily introduced to the study and to its purpose in detail. Consent form was signed before the date and time were set for the interview. The date and time for an interview was moreover at the convenience of both the researcher and the selected participants.

By history, taking during data collection such as the variety of causes or unmodifiable risk factors i.e. hereditary, genetic developmental reactions, auto-metabolic degradation and mechanical deficits may help the researchers assess the loss of cartilage strength leading to an inflammation of the osteons that affects their QOL (Marc et al. 1995).

The participants were reminded that the interview will be audio-recorded (using mobile phones) and reassured them that private and confidential information that were culturally sensitive and offensive to the Malaysian community would not be disclosed to the public. The data collected using a voice recorder uploaded in an external disc was kept safe in a locker as an ethical consideration.

All information collected was treated as confidential and the patient's identity was kept anonymous.

Detail of instruments. A hand-held voice recorder found in a mobile phone device was used to record with up to 60 minutes of interview session. These were then transcribed

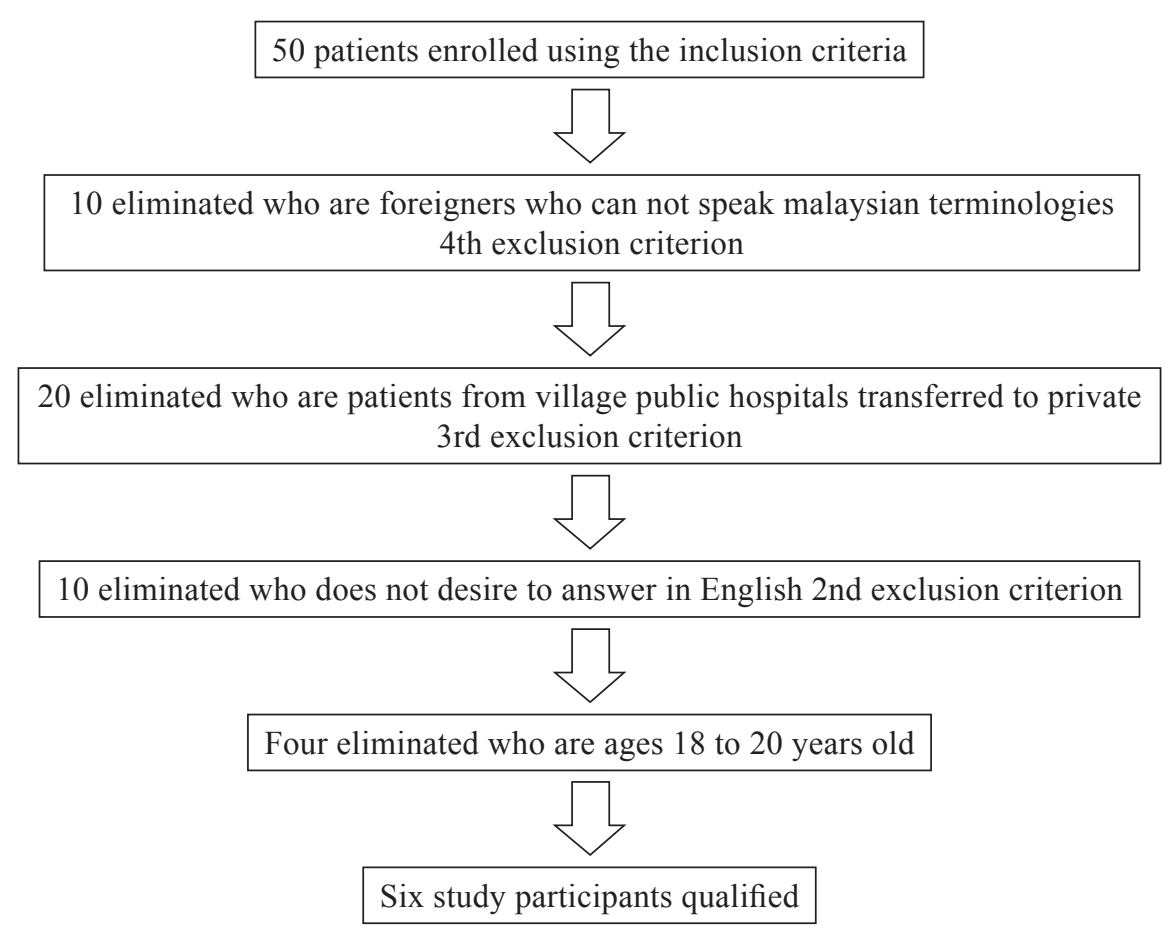

Figure 2. How the six participants were selected. 
in verbatim by playing, fast-forwarding or rewinding the interview session. Recording answers from the enrolled participants will add value to the reliability of the data analysis since the researcher has evidences to prove its findings (Groves et al. 2009). The transcripts were then analysed related to the themes explained in the discussion section.

Detail of the questionnaires as a guide for the interview session. The domains found on Figure 1 are the themes that addressed the questions.

The questions involved were: (1) physical capacity before and after physiotherapy, addressing the physiologic domain; (2) Effect on the emotional status and social activities, addressing the psychological domain; (3) Degree of difficulty and its effect on work performance, addressing the environmental domain; (4) Severity of disease before and after rehabilitation, addressing the level of independence domain; (5) Adaptations on how to cope with OA in their social life, addressing the social domain; (6) Knowledge on spiritual management, addressing the spiritual domain and (7) Impact of physiotherapy on their ability to financially cope with their QOL, addressing the financial domain.

In the Malay language it was translated as (1) Keupayaan fizikal sebelum dan selepas fisioterapi. The subjects would be further asked in a rephrased sentence Adakah anda mempunyai masalah-masalah lain yang mempunyai kekuatan fizikal anda, next is (2) Kesan ke atas status emosi dan aktiviti sosial, (3) Tahap kesukaran dan kesannya ke atas prestasi kerja, (4) Keterukan penyakit sebelum dan selepas pemulihan, (5) Penyesuaian pada bagaimana untuk menghadapi OA dalam kehidupan sosial mereka, (6) Pengetahuan mengenai pengurusan rohani, (7) Kesan fisioterapi kepada keupayaan mereka untuk kewangan menghadapi QOL mereka.

\section{Data Analysis}

The interviews were transcribed in verbatim and reread to ensure they were in a flow and focused on the themes - the domains of the QOL. The data analysis was counterchecked by two people who were not involved in the study but were experts in the analysis of the domains of the QOL. Where any differences arose, they were resolved by discussion and re-interpreted. Once transcribed, the transcripts were then red repeatedly before being coded to find emerging themes and issues, only then would it be finally written in the discussion section.

A thematic content analysis was then conducted with an open coding system in order to accommodate emerging themes and issues. Member-checking was also used to ensure higher validity. This was where the participant was called in to check the transcript to ensure it was transcribed and coded correctly in English according to how the selected participants for this research wanted it analysed. If the English wordings were incorrect as confirmed by the subjects (participants), it would then be revised by the researcher and re-checked by the selected participants for final confirmation. Once it was confirmed to be correct, only then could the answers be written in the discussion section.

\section{Ethical Considerations}

Ethical approval from the private-owned hospital in the state of Kuala Lumpur was sought before the study commenced. The researchers first had to register as a member of the National Medical Research Registry; then the study is registered with the Medical Research and Ethics Committee who reviewed the project and provided approval. The study was also approved by the university research governance and ethics committee.

To ensure internal and external validity and reliability of the study, the data was collected and held confidentially and stored securely. 


\section{FINDINGS}

The rehabilitation and physiotherapy conducted among six patients in the private hospital located in peninsular Malaysia explored QOL on the level of independence, environmental and psychological domain as compared with physiological, social, spiritual and financial domains with lesser responses. There were phenomenological experiences found among the six patients during the interview sessions.

Of the six patients interviewed, one was a staff nurse, aged 46 years old female with gardening as a hobby. Another was a 43 year old male sports trainer. The third one is a public health nurse, a 50 years old female who likes dancing as a hobby. The fourth one is a 41 year old female social worker, who likes to read books. The fifth patient interviewed is 51 years old assistant male nurse who likes jogging. Lastly, the sixth patient interviewed is a female dentist who is 50 years old and likes dancing.

Of the six patients interviewed there were four females and two males who had OAs. The diversity of their job experiences were two nurses, a sports trainer, a social worker, a dentist and a physiotherapist.

\section{Environmental Domain Affecting the QOL Before Rehabilitation}

Four respondents explored their lived experiences with physiotherapy and rehabilitation as it affects the environmental domain of their QOL. The two other respondents had no comments on this specific domain.

Before physiotherapy, a 46 year old female staff nurse said, "I've difficulty going up and down stairs every morning. I've hold out stairs railing while going up." She added that in order to cope "I often use lift in my working place and avoid frequent movement like sitting, standing or squatting."
A 43 year old male sports trainer said that before physiotherapy "it doesn't affect my working performance actually because my nature of work does not need to stand too long. However due to my knee problem, I'm less confident to play badminton too long."

A 51 year old male assistant nurse before physiotherapy said " $m y$ work requires doing a lot of walking in the ward but it has been affecting my working performance due to the pain."

A 50 year dentist before physiotherapy session said " $m y$ work requires doing a lot of walking in the ward but it has been affecting my working performance due to the pain."

\section{Physiologic Domain Affecting the QOL After Rehabilitation}

Of the six respondents, only five explored their lived experiences with physiotherapy and rehabilitation as it affected the physiologic domain of their QOL.

The QOL found from a 50 years old female dentist affecting her physiologic domain said that she "eats calcium tablets and drink milk to prevent joint pain. I also do exercises and cycling to improve muscle strength".

Another answer coming from a 51 years old assistant male nurse was "severe pain, difficulty to gets up from bed in the morning, but still can be tolerate. I felt mild stiffness at my knee joint but it becomes less after doing a couple of warming up exercises on the bed".

The physiologic domain again was emphasized when interviewing a 41 year old female social worker as she said that "pain has reduced and muscle power also improved after doing the following exercises such as the foot massager and attend reflexology to relieve 
pain. I also do some simple exercises taught by physiotherapist at home."

While the 46 years old female staff nurse said that "I used hot pack to relieve pain. I also do some simple exercises to feel healthy. I feel much better after doing some exercises, pain has reduced."

According to a 43 years old male sports trainer after rehabilitation said that "Ifeel much better now, in terms of pain and my capacity to play improved".

\section{Social Domain Affecting the QOL After Rehabilitation}

Of the six interviewees, only four explored their lived experiences with physiotherapy and rehabilitation as it affected the social domain of their QOL.

After rehabilitation, a 43 year old male sports trainer said that his social domain was affected as he said "think well, generally, in term of quality of life. The only thing is due to my knee pain, I can't join my friends go for badminton too often. I feel my life is so boring especially during weekends." However, he added a disappointment that he "always become emotional and disappointed if anyone talks about my joint disease".

A 41 year old female social worker on the other hand said that "I feel very angry and mentally disturbed. I also always argue with my husband and family members."

The 51 years old assistant male nurse after physiotherapy sessions said that he was "still able to attend social activities, on and off."

A 46 years old female staff nurse after physiotherapy said that "sometimes I feel frustrated, because I have to stop and be careful with my step in the garden. Every time I want to join any social activities, I've to think how the activity is going to be held." Whenever she socialized with other people she also added that "I'm always being emotional and disappointed if anyone talks about joint disease".

While the patients were socially incomplete, their QOL was achieved because of the spiritual domain. It was found that this domain was also affected after the physiotherapy sessions.

\section{Spiritual Domain Affecting the QOL After Rehabilitation}

Of the six, only two respondents explored their lived experiences with physiotherapy and rehabilitation as it affected the environmental domain of their QOL.

A 51 year old assistant male nurse after rehabilitation therapy said that "first I accept the fact that I am getting old and already will die soon. So I just accept the fact, I always have to be happy and enjoy my life as usual. Like do whatever I can do, if there is a pain just rest and continue my work and pray."

While a 50 year female dentist after physiotherapy sessions said "sometimes I blame myself for having OA, but I just pray because I know that the therapy works".

In order to be successful with their experience with physiotherapy sessions, it was also necessary to psychologically prepare themselves. It was found that this domain affected their QOL before and after the session.

\section{Psychological Domain Affecting the QOL After Rehabilitation}

Six respondents explored their lived experiences with physiotherapy and rehabilitation as it affected the psychological domain of their QOL. 
A 41 year old female social worker said that "My confidence level has improved. I became psychologically prepared to continue the exercises until end of my life. I know very well about this condition because I used to see many patients with this following condition but after attended Physiotherapy treatment I got to know more information about this problem." She added that "I learn many new things about managing knee pain from the Physiotherapist." With regards to the impact in attending physiotherapy she said that "I have learned how to overcome the pain and joint problems. I am also confident and able to tolerate the pain. Now I am able to walk with less pain without limping."

A 51 year old assistant male nurse says that before attending the physiotherapy sessions his psychological capacity was "not much. I only knew about osteoporosis and gout." While the psychological impact that the physiotherapy had given him was "very good. I get more confidence in managing my knee arthritis. It's good for me and helps me a lot. Thanks to the Physiotherapist to help and guided me in all aspects." Lastly, the physiotherapy affecting his psychological life in general "improves my confidence level because I understand that my knee pain is not a life threatening disease. It can be improved or maintained."

A 50 year old female dentist before attending the physiotherapy sessions said that "my psychological confidence was not much; all I know is my joint had wear and tear which causes pain." She added that "before attending physiotherapy, I have little confidence about managing joint problem. After attending physiotherapy, the physiotherapist gives more explanation and advice about my problem. Now, I understand what knee osteoarthritis is and how to manage the problem. My psychological point of view has changed and improved." However, she said that one of the limitations after attending physiotherapy sessions was "to stay home and it is sometimes psychologically annoying".

A 50 year old public health female nurse said that before attending physiotherapy her psychological confidence in managing pain was "not much, all I know is my joint is rubbing somewhere and cause pain."

The psychological impact to a 43 year old male sports trainer was "my depression level has gone down. At least I know this is not a life threatening disease." Another psychological impact in general he added was "after attending physiotherapy rehab, I know how to manage pain associated with prolong standing or walking. I knew when to sit and rest before the pain comes. For long distance walk, I will bring my walking stick along with me."

While the psychological impact to a 46 year old female staff nurse after experiencing physiotherapy said "I am not afraid about the knee condition, because now I have better understanding about the following problem and it is only related to my knee and not affect any other part of my body." She also added that generally, "I am happy that I am able to cope with the problem."

These psychological, spiritual, social and most of all the physiologic domains were found to have affected their independent performances of daily activities.

\section{Level of Independence Domain Affecting the QOL After Rehabilitation}

Six respondents explored their lived experiences with physiotherapy and rehabilitation as it affects the level of independence domain of their QOL.

After rehabilitation, a 41 year old female social worker said "every time I took painkiller to relief pain after few sessions of treatment I felt 
the pain reduced. My knee joint became flexible and easy to walk even for long distances it was really suffering especially in the morning and while walking before treatment. Physiotherapy really works for me." She added that the limitations however, was that "I've difficulty going up and down the van during home visits. I've to hold the seat and sometime I have to kneel on the van to get down." In order to adapt or cope with the situation, she said "I prefer to walk less and allotted my work to juniors. I always does sitting job."

A 51 year old male assistant nurse commented on the limits after physiotherapy sessions and said that my "fear of fall, scared my superiors. I use stick to balance my walking." That is why he says that "I wear knee guards while walking."

A 50 year old female dentist after sometime of rehabilitation process said "actually the pain is not so bad. But I feel there is joint stiffness and my knee movements are restricted."

A 46 year old female staff nurse after physiotherapy said "it was really hurt when I walk on uneven surfaces especially on roads before treatment. I felt more pain in the morning while getting up from the bed. I shake my knees to relieve the pain. After few sessions of treatment I feel the pain is reduced and a bit easy to walk." With regards to its effect on her work performance, she said "I was very careful during my knee movements and little bit slowly in working."

While a 43 year old male sports trainer said that in order to adapt or cope with his activities "every 20-30 minutes I need rest because my game needs to move a lot."

A 50 year old female public health nurse said that the limits that affect working life were that "I become scared of falling, so I use walking stick for support." She added that in order to adapt or cope with her OA after rehabilitation she "ignores the pain, and sometimes do less work." However, the physiotherapy sessions impact her life and say "pain is much relieved, as I am able to walk longer distances, knee joint much stable and no need to use walking stick."

It was also found that financial domains have a significant impact after physiotherapy sessions were conducted among these patients with OAs.

\section{Financial Domain Affecting the QOL After Rehabilitation}

Of the six respondents, only two respondents explored their lived experiences with physiotherapy and rehabilitation as it affected the environmental domain of their QOL.

A 50 year old female public health nurse said that "most of the time I take leave without pay from work and prefer to stay home."

On the contrary a 50 year old dentist said that "it is not much affecting my working life and my salary is not affected." However, she added that "in order to ignore the pain enjoying and buying food to eat is the expensive solution."

\section{DISCUSSIONS}

These six participants were interviewed using a standardized interview schedule which helped the researcher to remain in control of the interview and to ensure higher validity and reliability of the study findings. Internal and external validity and reliability was also ensured through member checks, informed consent and verbatim transcription of the interviews. The study also received ethical approval from Teesside University and private owned hospital in Kuala Lumpur.

The strength of this study was its design - qualitative phenomenological. The lived 
experiences of the respondents were very essential in analyzing the qualitative aspect of the domains of the QOL after rehabilitation from OAs.

However, the weakness of this study was its limitation which is (1) The medium of communication and (2) The number of population having only six participants. If the population were saturated (quantitative), it would have a quantified interpretation of the results. And if it were also conducted in Bahasa Melayu, interpretation of the questions might be easier for the interviewees to answer.

Most of the time, the questions were clear but the answers were different which was why it was necessary to repeat the same question until the authors (researchers) were satisfied that the answer was appropriate to be used for data analysis.

The following sections discusshow the data was analyzed.

\section{Thematic Analysis}

The questions set for interview were divided into themes addressing the seven domains of the QOL.

Effect on the emotional status and social activities. This theme addressed the psychological domain of the QOL. Most of the participants said that their knowledge was more likely to correlate with how they perceived and thought psychologically. This theme explored on how the patients with OAs felt about their capability to understand their diseases which also gave them emotional encouragement. However, their answers sometimes led to social domain as they began to inject stories such as how they shared the knowledge with other friends and how they socially debated regarding their understanding with OA.
However, the researchers were able to use social issues on the next theme which was the effect on emotional status and social activities.

Adaptations on How to Cope with $O A$ in their social life. This theme addressed the social domain of the QOL. Most of the participants said that the social capacity to socially debate on OA was a form of developing their selfesteem. As they were aware of their illness, after rehabilitation they became more confident in telling their friends about how they felt whenever they went out and socialized with them. This domain was phenomenologically leading the participants to a QOL.

However, some of the participants still mislead their answers to how many times they filed their sick leaves and how many times they missed work. This was a different issue but nevertheless, it was also a part of the themes set for interview called the degree of difficulty and its effect on work performance.

Impact of physiotherapy on their ability to financially cope with their QOL. This theme addressed the financial domain of the QOL. Most of the interviewee said that because of physiotheraphy their financial statuses were being compromised. This was because they tended to overuse their sick and annual leave as the need to be operated arose some physicians advised then to be on complete bed rest. If they began their leave "without-pay", then their budget to pay the hospital bills would also be compromised. Since they cannot work for a living, they just had to face this specific degree of difficulty.

One of the participants said that her financial needs in addition to their physiotherapy sessions were adequate. Even though medications were very expensive and it was bought on a monthly basis, some were able to afford. This 
was because the subjects were earning about RM 3000 monthly.

According to Deutsch et al. (2003), both poverty and inequality of standard of living and having more than the basic salary rate does not necessarily lead to a QOL. However, two of the six participants with more than the basic monthly earnings phenomenologically achieved the financial domain of the QOL.

The focus of the conversation was led directly to the next theme called physiologic domain since the harm of losing money could be outweighed by the physiological benefits of physiotherapy.

Physical capacity before and after physiotherapy. This theme addressed the physiologic domain of the QOL. Most of the participants answered the questions that focus on their physiologic functioning and how strong they were before they acquired the disease called OA. The authors had also explored the severity of their disease before and after the rehabilitation and physiotheraphy programme - how they began to regain their strengths and became better. However, the Malaysian culture affected the physiotherapy's purpose, one way or the other, in achieving their physiologic domains leading to a QOL.

A participant did foot massage from the blind; and one practiced an internal Chinese martial art exercise called Taichi. These two Malaysian cultures were neither indicated nor contraindicated to physiotherapy. However, it had phenomenologically led to a QOL in the physiologic domain.

Most of them began to be emotional and spiritual in expressing their answers. The discussion on the spiritual domain of patients in their general life was the next question.
Knowledge on spiritual management. This addressed the emotional and spiritual domain of the QOL. Only two subjects were able to answer the question appropriate to the domain which discussed forgiveness and blamed affecting their spirituality and emotions. The two subjects were exploring their emotions on how they blamed themselves for having OA and how they forgave others who contributed to their disease because they knew that their physiotherapy sessions would phenomenologically soon be successful, because of prayers.

However, there was a thin dividing line between the spiritual and psychological domain of the QOL. Most of the subjects' answered to the psychological fall on the spiritual domain.

Severity of disease before and after rehabilitation. This theme addressed the level of independence domain of the QOL. Most of the participants said that they had phenomenologically achieved to acquire an independent self-management because of the handbooks that were given to them by the physiotherapists. It included self-drug administration and first aid care for their OA. Most of the participants were happy when they began to be independent in taking care of themselves.

Degree of difficulty and its effect on work performance. This addressed environmental domain which might also have effect on the financial domain and level of independence.

After physiotherapy and rehabilitation sessions, most of the participants said that they began to feel safe with their environment. They acquired courage to participate in rigorous activities with friends as they believed that they could manage to cope with an unpleasant environment. Even the environment at their workplace was becoming more conducive to them and they tended to lessen their sick leaves. 
Overall, none of the subjects with OA were able to focus their answers to the questions set. All of them diverted from one topic to another. Nevertheless, the author was able to use their answers and place it on the appropriate domain for interpretation of the findings.

Discussing the findings was important in order for it to be implied into practice.

\section{Implications to Practice}

In applied physiotherapy, it is important to address age, gender and hobbies before implementing physiotherapy and rehabilitation sessions among patients with OAs.

Age is correlated with the application of physical exercises as it is expected that muscular and skeletal system degenerates as humans grow older (Tortora \& Derrikson 2010). In addition, gender must also be considered when dealing with patients with OAs. This is because the female gender is more prone to skeletal degeneration as calcium in their body is decreased especially in menopausal age (Marc et al. 1995). This menopause syndrome decreases bone strengths because estrogen is also decreasing in production. Thereby decreases calcium production as an effect of decreasing estrogen production (Tortora and Derrickson 2010). This decrease in calcium will be exacerbated by multiple pregnancies (Felson 2004). The more pregnancies a female gender experiences, the more calcium they will lose as they grow older (Tortora \& Derrickson 2010).

The male gender however, is not prone for decreasing calcium but rather by the activities or hobbies which they are inclined to (Tortora $\&$ Derrickson 2010). Which is why in applied physiotherapy, it is important to ask about the hobbies of the patients (Marc et al. 1995). Repetitive disorders such as tendonitis as the effects of hobbies are co-morbidities of OAs (Jakobsson \& Halber 2002). This information is necessary to be asked by physiotherapists among their patients with OA that are for rehabilitation (Morden et al. 2014; Fujita et al. 2006; Roos \& Larsen 2003).

\section{CONCLUSION}

Phenomenologically, physiotherapy and rehabilitation helped OA patients achieve a QOL. However, in this study, not all the domains of the QOL are phenomenologically achieved. This was because the study participants had their own perceptions and points-of-views on describing their lived experiences with physiotherapy the most of the time did not require the seven domains of the QOL.

In addition, the answers of the study participants to the interviews given might have integrated some of the domains such as the psychological, spiritual and social domains or the physiological, environmental and level of independence domains in one of their answers.

That is why it was recommended that in future research, a mixed design could be conducted so that triangulation of both quantitative and qualitative findings could strengthen the discussion and the conclusion.

\section{ACKNOWLEDGEMENT}

Special thanks is acknowledged to Teesside University for the supervision given. Many thanks as well to Edu Punay for editing this final written research.

Date of submission: December 2015

Date of acceptance: January 2016 


\section{REFERENCES}

Abdullah, A, Reem, A, Ehab, E, Dia, S \& Shaima, A 2013, 'Attitudes to knee osteoarthritis and total knee replacement in Arab women: a qualitative study', BMC Research Notes, vol. 2013, no. 6, pp. 406.

Blixen, C \& Kippes, C 1999, 'Depression, social support and quality of life among elderly with osteoarthritis', Journal of Nursing Scholarship, vol. 31, no. 3, pp. 221-222.

Bosomworth, N 2009, 'Exercise and knee osteoarthritis: benefit or hazard?', Canadian Family Physician, vol. 55, pp. 871-878.

Brooks, P 2002, 'Impact of osteoarthritis on individuals and society: how much disability? Social consequences and health economic implications', Curr. Opin. Rheumatol, vol. 14, 573-577.

Coggon, D, Reading, I, Croft, P, Mclaren, M, Barrett, D \& Cooper, C 2001, 'Knee osteoarthritis and obesity', International Journal of Obesity, vol. $25,622-627$.

Chin C, Rabia K, Mohazmi M, Nik S, Ng C, 2012, 'Evidence for persisting with treatment with paracetamol in patients with mild to moderate osteoarthritis of the knees', Life Science Journal, vol. 9 , no. 3 .

Denzin, K \& Lincoln, Y 2005, The sage handbook of qualitative research, Sage publications, CA.

Deutsch, J, Ramos, X \& Silbe, J 2003, 'Poverty and inequality of standard of living and quality of life in Great Britain', in Advances in quality of life theory and research, eds Sirgy et al., Kluwer Academic Publishers, The Netherlands, pp. 99-128.

Dias, R, Dias, J, \& Ramos, 1 2003, 'Impact of an exercise and walking protocol on quality of life for elderly people with OA of the knee', Physiotherapy Research International, vol. 8, no. 3, pp.121-130.

Fallowfield, 1 (2009), 'What is quality of life?', viewed 28 May 2013, <http://www.medicine. ox.ac.uk/bandolier/painres/download/whatis/ whatisqol.pdf $>$.

Felson, D 2004, 'An update on the pathogenesis and epidemiology of osteoarthritis', Radiol. Clin. North Am., vol. 42, pp.1-9.
Fujita, K, Kiyoko, M \& Takao, H 2006, 'Qualitative study of osteoarthritis patients' experience before and after total hip arthroplasty in Japan', Nursing and Health Sciences, vol. 8, pp. 81-87.

Groves, R, Floyd J, Fowler, J, Couper, M, Lepkowski, J, Singer, E \& Tourangeau, R 2009, Survey methodology, John Wiley \& Sons, New York.

Jakobsson, U \& Hallber, I 2002, 'Pain and quality of life among older people with rheumatoid arthritis and/or osteoarthritis: a literature review', Journal of Clinical Nursing, vol. 11, pp. 430-443.

Li, C, Rubini, P, Anthony A, Victoria A, Mohamed B, Raman, M, Gavinn, N \& Mohit, B 2013, 'Is the treatment gap in knee osteoarthritis real? A qualitative study of surgeons' perceptions', Journal of Long-term Effects of Medical Implants, vol. 23, no. 2-3, pp. 223-240.

Marc, C, Roy, D, Kenneth, D, Bruce, M, Paul, A, Marie, R, Rosland, M, \& Thomas, J 1995, 'Guidelines for the medical management of osteoarthritis', Journal of the American College of Rheumatology, pp.1541-1546.

Ministry of Higher Education 2010, 'Malaysian education 2010-2015', viewed 12 August 2015, $<$ http://www.moe.gov.my/en/home> .

Morden, A, Clare J, Bie, O, Porcheret, M \& Dziedzic, K 2014, 'Acceptability of a 'guidebook' for the management of osteoarthritis: a qualitative study of patient and clinician's perspectives', $B M C$ Musculoskeletal Disorders, vol. 15, pp. 427.

Power, D, Elizabeth B, Melissa R, Angela J \& Gillian H 2008, 'Fatigue in osteoarthritis: a qualitative study', BMC musculoskeletal disorders, vol. 9, no. 63 .

Roos, E \& Larsen, S 2003, 'Knee injury and osteoarthritis outcome score (KOOS) validation and comparison to the woman in total knee replacement', Health and Quality of Life Outcomes, vol. 1, pp. 1-10.

Rosemann, T, Grol, R, Herman, K, Wensing, M, \& Szecsenyi, J 2008, 'Association between obesity, quality of life, physical activity and health service utilization in primary care patients with osteoarthritis', International Journal of Behavioral Nutrition and Physical Activity, vol. 5 , no. 4 .

Rosemann, T, Korner, T, Wensing, M, Gensichen, J, Muth, C, Joos, S \& Szecsenyi, J 2005, 'Rationale, 
design and conduct of a comprehensive evaluation of a primary care based intervention to improve the quality of life of osteoarthritis patients. the praxart-project: a cluster randomized controlled trial', [isrctn87252339], BMC Public Health, vol. 5, pp. 77.

Tortora, G \& Derrikson, B 2010, Priniciples of anatomy and physiology, 12th edn, Wiley and Sons, New Jersey.

Saldana, J 2012, The coding manual for qualitative researchers, Thousand Oaks, Sage Publications, CA.
Wolfey, F \& Holley, D 1997, 'Measurement of the quality of life in rheumatic disorders using the EuroQol', British Journal of Rheumatology, vol. 88 , no. 25 , pp. 675-682.

World Health Organization 1998, 'Quality of life', viewed, 1 August 2015, <www.who.com>.

Zakaria, Z, Azman bakar, Hadzri Hasmoni, Fauzi Rani \& Samiah Kadir, 2009, 'Health-related quality of life in patients with knee osteoarthritis attending two primary care clinics in Malaysia: a cross-sectional study', Asia Pacific Family Medicine, vol. 8, no.10. 